\title{
Extremely-Wide-Range Supply-Independent CMOS Voltage References for Telemetry-Powering Applications
}

\author{
AMIR M. SODAGAR ${ }^{1,2}$ AND KHALIL NAJAFI ${ }^{2}$ \\ ${ }^{1}$ Electrical Engineering Department, K.N. Toosi University of Technology, Tehran, Iran \\ ${ }^{2}$ Center for Wireless Integrated MicroSystems, University of Michigan, Ann Arbor, MI, USA \\ E-mail:asodagar@umich.edu;najafi@umich.edu
}

Received February 12, 2004; Revised January 31, 2005; Accepted April 21, 2005

\begin{abstract}
This paper reports a voltage reference circuit in standard CMOS process. It exhibits excellent supply independency for a wide input voltage range, which is of great importance in telemetry-powered systems. This circuit is based on the well-known $V_{G S}$-reference supply-independent current reference circuit, but it is designed to serve as a voltage reference. While the reference current generated by this circuit varies with the supply voltage, a selfcompensating mechanism can be found in voltage-mode operation of the circuit that results in a supply-independent reference voltage. This supply independency is well observed in the static operation of the circuit over an extremely wide input range, as well as in its dynamic behavior for high frequency ripples on the input voltage. Based on the proposed idea, a multi-output voltage reference and a CMOS DC level shifter are also designed. The proposed voltage reference circuits have been fabricated using MOSIS $1.6 \mu \mathrm{m}$ standard CMOS process. The basic voltage reference provides $957 \mu \mathrm{V} / \mathrm{V}$ static supply dependency, rejects input ripples of up to $8 \mathrm{MHz}$ by $60 \pm 3 \mathrm{~dB}$, and consumes only $15.8-36.9 \mu \mathrm{A}$ when the input voltage varies in the range $2.6-12 \mathrm{~V}$.
\end{abstract}

Key Words: voltage reference, DC level shifter, supply-independent circuits

\section{Introduction}

Telemetry powering is of interest in some advanced branches of technology like biomedical implantable micro-systems used for nerve stimulation, neuromuscular stimulation, and neural recording. Long-term and even lifelong operation without any replaceable energy source (e.g. batteries), ease of movement without being restricted by power wires, and avoiding the risk of infection and patient discomfort caused by percutaneous wires, necessitate the biomedical implantable microsystems to wirelessly communicate and be energized [1-5]. To realize this idea, the energy required for the operation of such systems is transferred from an external source to the implanted system by using an external transmitter and an internal receiver. The received waves are rectified and then regulated in order to provide the supply voltage for the implanted system. In such systems, because of transmitter or receiver movements, the distance or alignment between the transmitter and the receiver antennas varies, thus resulting in disturbances or variations in the induced voltage on the receiving antenna. To have a stable supply voltage in the presence of such input variations, the voltage regulator should be able to operate over a wide range of input voltages, and at the same time the output voltage should be highly input independent.
To serve as the voltage reference in the required voltage regulator, regular and Zener diodes have been employed in [2] and [3-5], respectively. But because of the non-negligible diode series resistance, the reference voltage would not be satisfactorily constant when the input voltage drastically changes.

Disregarding its temperature dependence, the $V_{G S^{-}}$ referenced supply-independent current reference circuit shown in Fig. 1 is a good reference circuit, although it shows much more supply dependency than its bipolar counterpart [6]. It is shown in the next section that there can be found a self-compensating mechanism in voltage-mode operation of the same circuit that can result in a stable reference voltage over an extremely wide range of input changes, provided that it is properly designed [7]. Based on this mechanism, a multi-output voltage reference [8] and also a DC level shifter will be proposed in the next sections.

\section{Circuit Concept and Design}

Assuming that $I_{R}$ is constant in the $V_{G S}$-referenced circuit of Fig. 1, then $V_{G S 1}$ and $V_{G S 2}$ are also constant. So, $V_{G S 1}+V_{G S 2}$ that appears at node A can be considered as a constant reference voltage:

$$
V_{\mathrm{REF}}=V_{G S 1}+V_{G S 2}
$$




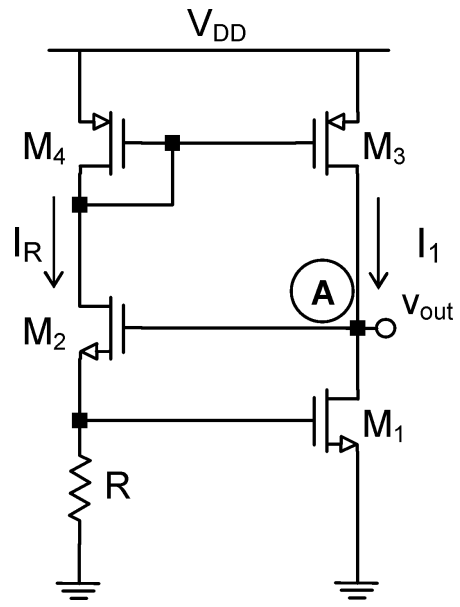

Fig. 1. $V_{G S}$-referenced supply-independent reference circuit.

But it is known that $I_{1}$, and to some extent $I_{R}$, vary with supply voltage and hence the above reference voltage tends to follow supply changes. When the supply voltage $\left(V_{D D}\right)$ is increased, $V_{S D 3}$ and consequently $I_{1}$ will increase. This causes $V_{G S 1}$ to increase with $V_{D D}^{1 / 2}$, which sets the current through $R\left(I_{R}\right)$. However it seems that this will cause $V_{G S 2}$ to increase with $V_{D D}^{1 / 4}$, but there is another factor that contributes to determining $V_{G S 2}$. Remembering that:

$$
V_{G S}=V_{T}+\left\{2 I_{D} / \beta\left(1+\lambda V_{D S}\right)\right\}^{1 / 2}
$$

$V_{D S 2}$ can play a second-order role. It can be seen in the circuit that $V_{D S 2}$ increases with $V_{D D}$. So, by properly setting the design parameters, it is shown that there can be found a situation that $V_{G S 2}$ is so decreasing that compensates the increase of $V_{G S 1}$, when $V_{D D}$ is increased, resulting in a supply-independent reference voltage.

Mathematically speaking, in order to achieve a supply-independent reference voltage, one should have:

$$
\delta V_{\mathrm{REF}} / \delta V_{D D}=0
$$

or:

$$
\delta V_{G S 2} / \delta V_{D D}=-\delta V_{G S 1} / \delta V_{D D}
$$

As is derived in the Appendix, solving device and circuit equations for the above relationship leads to the following optimization equation:

$$
\left(R \sqrt{2 I_{R}}\right) \cdot\left(\lambda_{N} \cdot \sqrt{b \cdot \beta_{1}}-\lambda_{P} \cdot \sqrt{a^{3} \beta_{2}}\right)=b \lambda_{N}+a \lambda_{P}
$$

which relates the proper $W / L$ ratios for $M 1$ and $M 2$ and resistance $R$, where $a=1+\lambda_{N}\left(V_{D D}-V_{\mathrm{REF}}\right)$ and $b=1+\lambda_{P}\left(V_{D D}-V_{\mathrm{REF}}\right)$.
It is obvious that the basic design is done for obtaining the reference voltage, by:

$$
V_{\mathrm{REF}}=2 V_{T N}+\sqrt{2 I}_{R} \cdot\left(\sqrt{b / \beta_{1}}+1 / \sqrt{ } \beta_{2}\right)
$$

Note that Eq. (6) is written neglecting body effect for $M 2$. The only impact of the body effect would be on the value of the reference voltage, not its supply independency. In order to keep the simplicity of design Eqs. (5) and (6), the designer can solve them in order to have a rough estimate for channel sizes of $M 1$ and $M 2$, which play the most important role in optimizing the circuit. Then, the channel sizes can be trimmed by simulation in order to optimize the circuit considering the body effect and other second-order factors.

As a case study, the circuit of Fig. 1 is optimized for a $1.6 \mathrm{~V}$ reference voltage using MOSIS $1.6 \mu \mathrm{m}$ standard CMOS process. However there are processes with smaller feature sizes available, the reason for choosing such a large feature size (the largest available through MOSIS) is as follows: In telemetry powering applications the received voltage at the input of the front-end is supposed to be high enough to result in a stable supply voltage, after considering all the voltage overheads for the rectifier, the voltage reference, and also the other blocks in the voltage regulator. So, the process with the largest possible feature size has been chosen in order to achieve the highest tolerable voltages for the transistors.

In this case, design parameters are determined to be as follow:

$$
\begin{aligned}
(W / L)_{1} & =80 \mu / 1.6 \mu \quad(W / L)_{2}=4 \mu / 1.6 \mu \\
(W / L)_{3,4} & =4 \mu / 1.6 \mu \quad R=70 \mathrm{k} \Omega
\end{aligned}
$$

Figure 2 illustrates the more important waveforms for the optimized case. As is expected, $V_{G S 2}$ decreases with increasing $V_{D D}$, exactly with the same rate that $V_{G S 1}$ increases, and the result is a flat $V_{\mathrm{REF}}$ over a wide supply voltage range.

Increasing $V_{D D}$ from its minimum acceptable value of about 2.4 up to $20 \mathrm{~V}$ leads to a maximum deviation of $9 \mathrm{mV}(511 \mu \mathrm{V} / \mathrm{V})$ from the $1.625-\mathrm{V}$ reference voltage. Over this wide supply voltage range, the current consumption of the circuit varies between 15 and $43 \mu \mathrm{A}$.

\subsection{Dynamic Behavior}

Although the DC transfer curve for the voltage reference circuit is quite flat for $V_{D D}$ greater than $V_{D D}(\mathrm{~min})$, it should be noted that it only shows static dependency of the reference voltage on $V_{D D}$ variations. However, ripples on the unregulated supply rail $\left(V_{D D}\right)$ appear in an $A C$ or dynamic nature. Simulations show that the circuit 

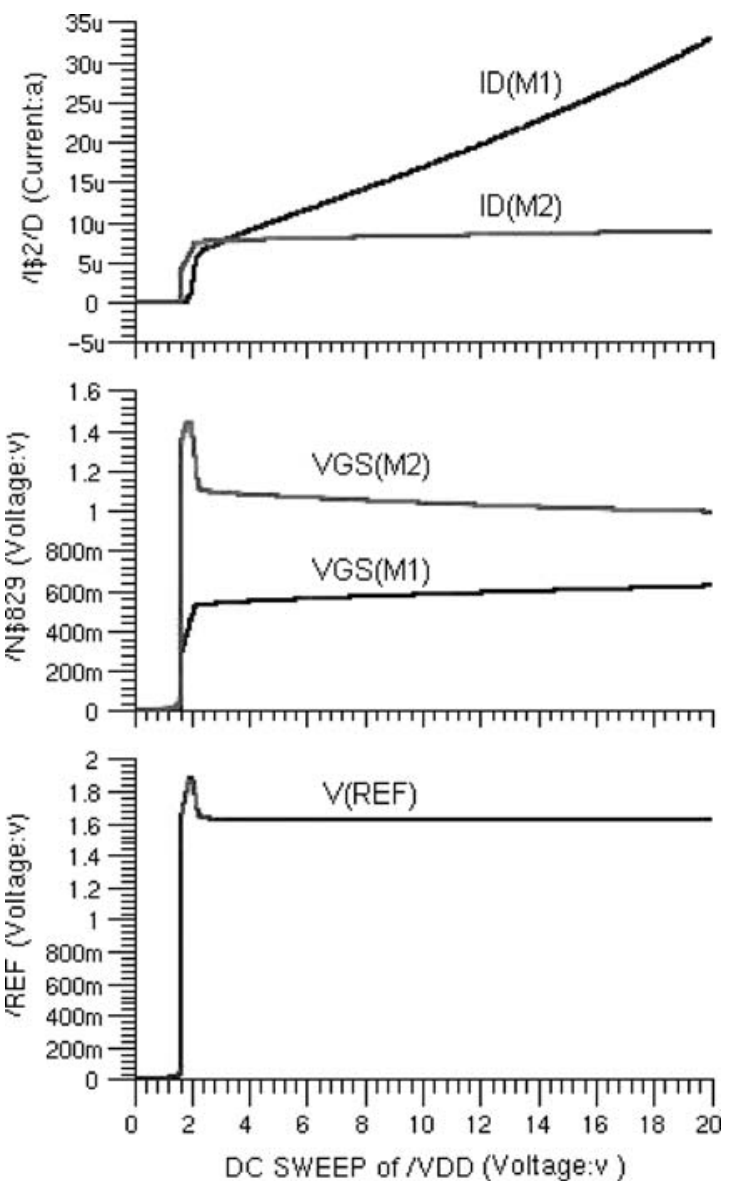

Fig. 2. Important currents and voltages in the optimized voltagereference circuit.

rejects ripples by about $-59 \mathrm{~dB}$ with $3 \mathrm{~dB}$ frequency of $1.5 \mathrm{MHz}$.

It is obvious that like many other supply dependent reference circuits, the circuit of Fig. 1 has two stable states and requires a startup circuit to guarantee its operation in the desired operating point.

\section{DC Level Shifter}

Theoretically, a voltage reference circuit generates a constant voltage difference between the output node and the ground. In another area of analog circuits there are DC level shifters whose functional behavior can be defined as providing a constant voltage difference between the output and the input nodes. Designing the complementary version of the circuit of Fig. 1 would result in an excellent CMOS DC level shifter, as is shown in Fig. 3.

Shown in Fig. 4 is the DC level shift as a function of the input voltage, which demonstrates excellent input independency over a very wide range of the input. The optimized behavior of the circuit in MOSIS $1.6 \mu \mathrm{m}$ standard CMOS process is achieved by the following

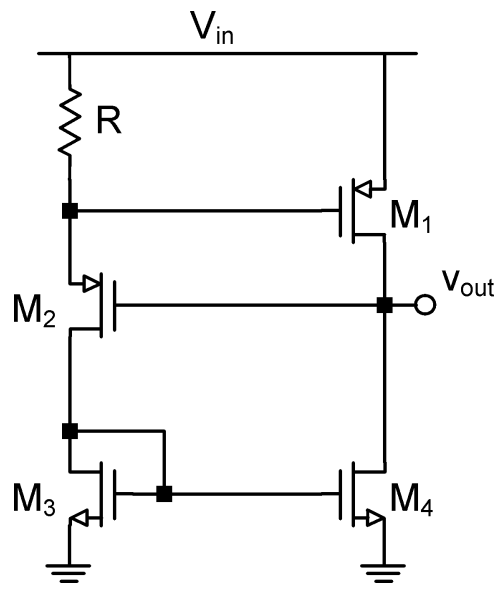

Fig. 3. Schematic of the proposed CMOS DC level shifter.

design parameters:

$$
\begin{aligned}
(W / L)_{1} & =80 \mu / 1.6 \mu \quad(W / L)_{2}=4 \mu / 4 \mu \\
(W / L)_{3,4} & =4 \mu / 1.6 \mu \quad R=70 \mathrm{k} \Omega
\end{aligned}
$$

In this case, the DC level shift is $3.081 \mathrm{~V} \pm 8 \mathrm{mV}$ when the input voltage varies from about 3.5 to $20 \mathrm{~V}$. This means that the DC level shift depends on the input voltage just by less than $0.5 \mathrm{mV} / \mathrm{V}$, and the circuit is well operating by an overhead voltage of as low as $0.42 \mathrm{~V}$ (in this case).

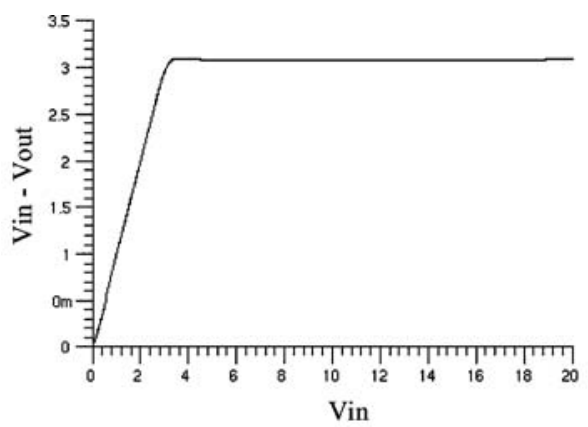

(a)

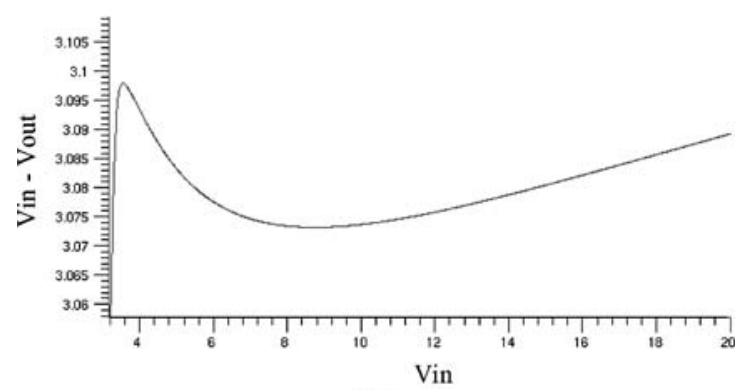

(b)

Fig. 4. DC level shift as a function of the input voltage (a) broad view and (b) close view. 


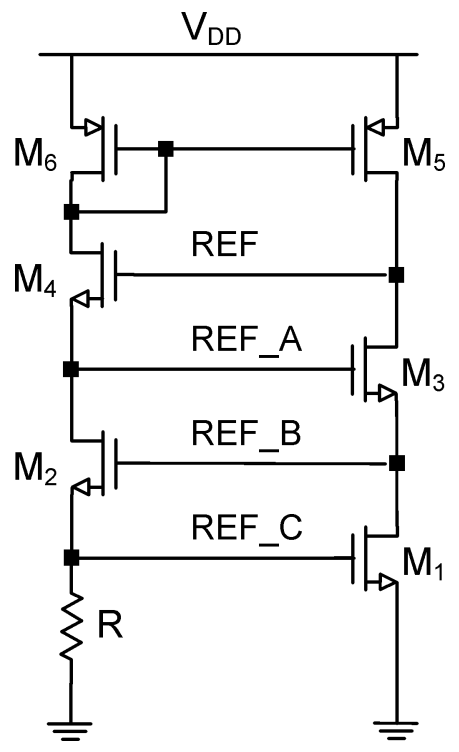

Fig. 5. Proposed multi-output voltage reference circuit.

\section{Multi-Output Voltage Reference Circuit}

As was described in Section 2, assuming that $I_{R}$ is constant in the $V_{G S}$-referenced circuit of Fig. 1 , as $V_{D D}$ increases, $I_{D 1}$ and $V_{G S 1}$ increase with $V_{D D}$ and $V_{D D}^{1 / 2}$, respectively. The key point for optimizing the circuit is that transistor $M 2$ should be so designed that its $V_{G S}$ should decrease with $V_{D D}^{1 / 2}$ with such a rate that compensates the increase of $V_{G S 1}$. So, the reference voltage $V_{\mathrm{REF}}=V_{G S 1}+V_{G S 2}$ would be a $V_{D D}$-independent voltage.

Now, if we stack more transistors, the reference voltage will be the summation of more $V_{G S}$ terms, and obviously at a higher voltage level. Another benefit is to achieve more reference voltages with lower values at the lower nodes. Figure 5 shows the proposed voltage reference circuit that realizes this idea. Like in the circuit of Fig. 1, the current in the right hand side of the proposed circuit increases with $V_{D D}$, and hence $V_{G S 1}$ and $V_{G S 3}$ increase with $V_{D D}^{1 / 2}$. Again, transistor $M 4$ can be so sized that compensate the accumulated increase with $V_{D D}^{1 / 2}$ caused by $M 1$ and $M 3$. The reason for such a behavior is that since the drain current and drain-source voltage of transistor $M 4$ are being increased, satisfying the characteristic equation:

$$
V_{G S}=V_{T}+\left\{2 I_{D} / \beta\left(1+\lambda V_{D S}\right)\right\}^{1 / 2}
$$

implies that there is no way for its $V_{G S}$ but decreasing.

As a case study, optimizing the circuit of Fig. 5 for a $4 \mathrm{~V}$ main reference voltage in MOSIS $1.6 \mu \mathrm{m}$ standard CMOS process will result in the following design parameters:

$$
\begin{aligned}
& (W / L)_{1,3}=80 \mu / 1.6 \mu \quad(W / L)_{2,4}=11.2 \mu / 10 \mu \\
& (W / L)_{5,6}=4 \mu / 4 \mu \quad R=70 \mathrm{k} \Omega
\end{aligned}
$$

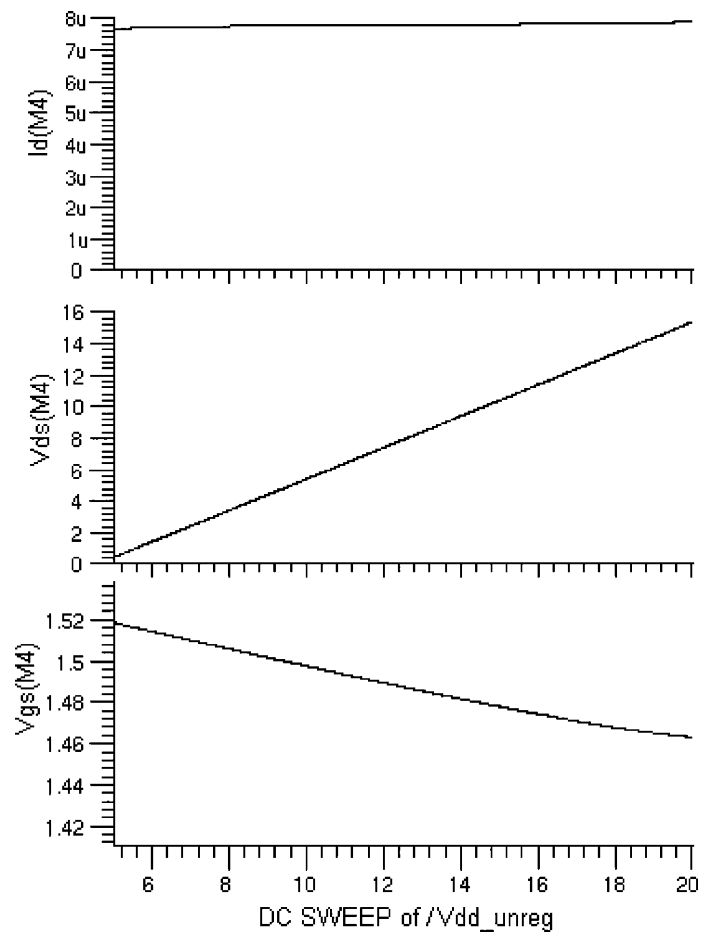

Fig. 6. $I_{D}, V_{D S}$, and $V_{G S}$ for $M 4$, as $V_{D D}$ increases.

In this case as $V_{D D}$ increases, $I_{D}, V_{D S}$, and $V_{G S}$ for M4 will be as shown in Fig. 6 .

As the result, Fig. 7(a) demonstrates how $V_{G S 4}$ has been capable of overcoming the accumulated increasing behavior of the three other gate-source voltages (i.e. $\left.V_{G S 1}+V_{G S 2}+V_{G S 3}\right)$. As the legends state, the traces are for $V_{\mathrm{REF}-\mathrm{C}}, V_{\mathrm{REF} \_B}, V_{\mathrm{REF} \_}$, and $V_{\mathrm{REF}}$, from bottom to up. It is a great achievement that over a $15-\mathrm{V}$ input range, from 5 to $20 \mathrm{~V}$, the increase in the reference voltages $V_{\mathrm{REF}_{-} \mathrm{C}}, V_{\mathrm{REF}_{-} \mathrm{B}}$, and $V_{\mathrm{REF} \_\mathrm{A}}$ is only 15,25 , and $40 \mathrm{mV}$, respectively, and the above-pointed self-compensating behavior limits the deviation in $V_{\mathrm{REF}}$ to less than $25 \mathrm{mV}$.

DC transfer curves for the four reference voltages, generated by the proposed circuit, are given in Fig. 7(b).

Simulations show that over the $15-\mathrm{V}$ input range, current consumption of the circuit varies between 15 and $17 \mathrm{uA}$.

\subsection{Dynamic Behavior}

Although the DC transfer curve for the voltage reference circuit is quite flat for $V_{D D}$ greater than $V_{D D}(\mathrm{~min})$, it should be noted that it only shows static dependency of the reference voltage on $V_{D D}$ variations. However, ripples on the unregulated supply rail $\left(V_{D D}\right)$ appear in an $A C$ or dynamic nature. Simulations show that the circuit rejects ripples by about -52.8 with $3 \mathrm{~dB}$ frequency of $10 \mathrm{MHz}$. 


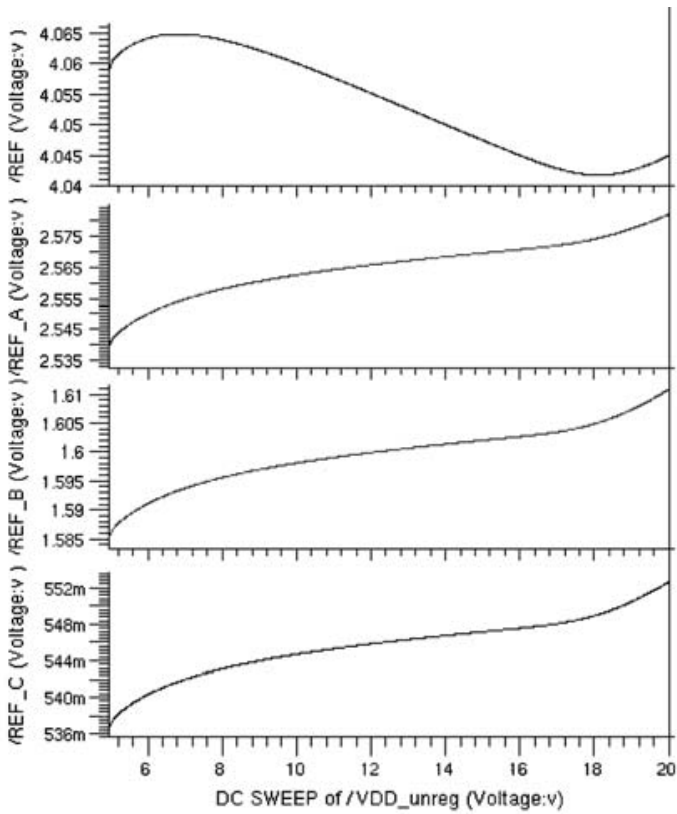

(a)

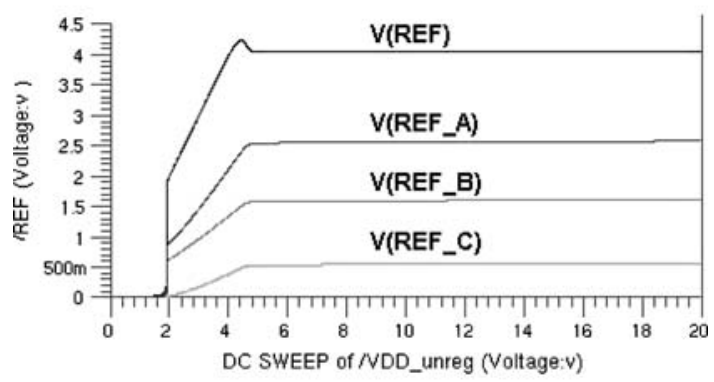

(b)

Fig. 7. (a) Close view, (b) broad view for the four generated reference voltages versus $V_{D D}$.

It is obvious that like many other supply dependent reference circuits, the proposed circuits have two stable states and require startup circuits to guarantee their operation in the desired operating point.

\section{Experimental Results}

\subsection{Simple Voltage Reference}

Shown in Fig. 8 is the photograph of the proposed circuit along with its startup circuit fabricated using MOSIS 1.6 um standard CMOS process.

Figure 9 shows the simulated and measured DC transfer curves of the optimized voltage reference circuit. As can be seen, because of a slight difference between transistor threshold voltages in simulation and in practice the output voltage level has been shifted up, but it is still extremely flat over a wide range of input from $2.6 \mathrm{~V}$ up to as high as $12 \mathrm{~V}$. This robustness (in the flatness of the inputoutput characteristic) against threshold voltage varia-

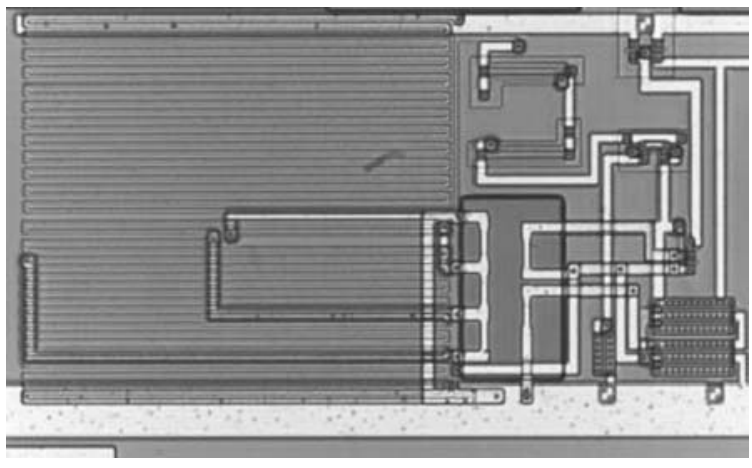

Fig. 8. Photo of the fabricated circuit along with its startup circuit.

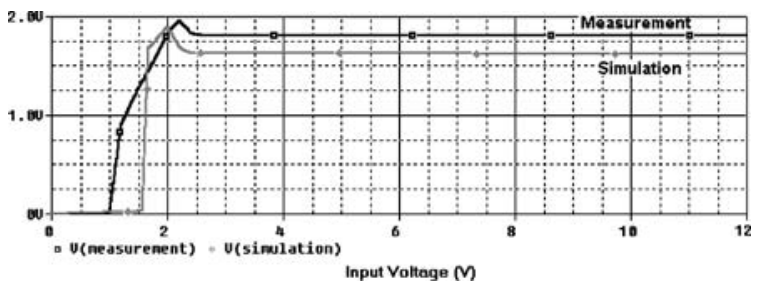

Fig. 9. Simulated and measured DC transfer curves for the circuit.

tions was predictable since transistor threshold voltages do not directly appear in the output flatness condition (Eq. (5)).

Although simulations show that the flatness of the DC transfer curve continues up to input voltages as high as $20 \mathrm{~V}$ (Fig. 2), but in practice the measurements were stopped by breakdown damages at about 10-12 V and the circuit couldn't be tested for higher voltages. However the transistors are not strong enough to tolerate very large voltages, but it should be emphasized that the optimization approach, which can be considered as the spirit of this paper, is strong enough to provide extremely robust reference voltages against very large line variations.

Figure 10 illustrates both simulated and measured Bode magnitude plots for ripple rejection ratio, which is the transfer gain from the input voltage to the generated reference voltage. The capacitance of the measuring

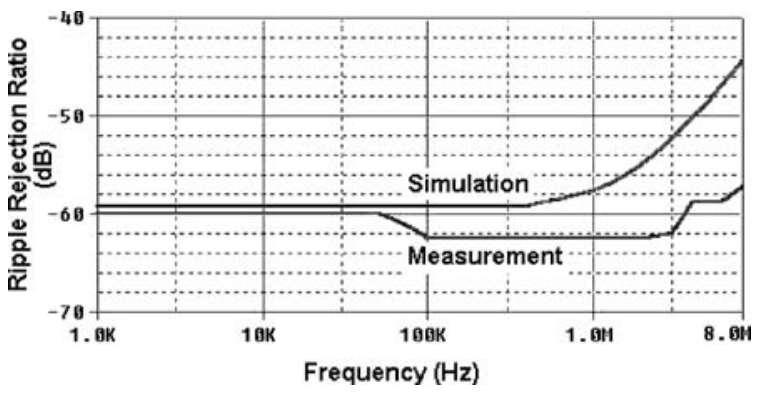

Fig. 10. Simulated and measured ripple rejection characteristic curves. 


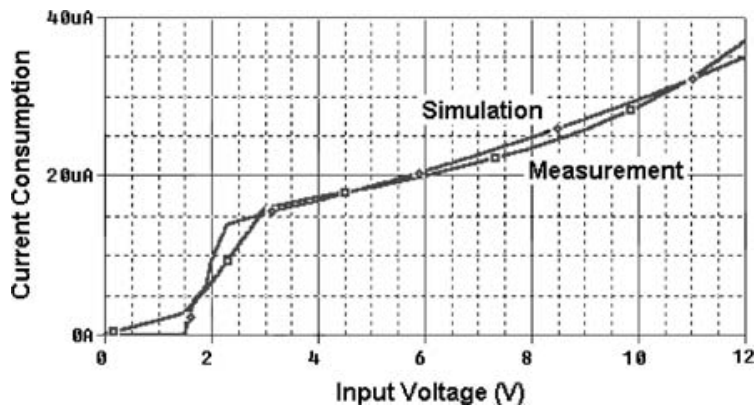

Fig. 11. Current consumed by the voltage reference and the startup circuits.

probe, when connected to the high-resistance output node, is the reason for achieving the measured ripple rejection curve better than the simulated one. Ripple rejection ratio for the fabricated circuit, which is a measure of its dynamic supply dependency, is within $-60 \pm 3 \mathrm{~dB}$ for frequencies up to $8 \mathrm{MHz}$. This is far larger and also is valid for much wider frequency range than that of the commonly reported CMOS voltage references.

Shown in Fig. 11 are the simulated and measured current consumptions for the optimized voltage reference circuit and its startup circuit, as a function of the input supply voltage.

Table 1 summarizes the simulated and measured specifications of the voltage reference circuit, which

Table 1. Simulated and measured specifications of the simple voltage reference circuit.

\begin{tabular}{lcc}
\hline Specification & Simulated & Measured \\
\hline Input voltage range & $2.4-20 \mathrm{~V}$ & $2.6-12 \mathrm{~V}^{*}$ \\
Static supply & $511 \mu \mathrm{V} / \mathrm{V}$ & $957 \mathrm{uV} / \mathrm{V}$ \\
$\quad$ dependency (DC) & $-59.2 \mathrm{~dB}$ & $-60 \mathrm{~dB}$ \\
$\begin{array}{l}\text { Dynamic supply } \\
\text { dependency(Ripple reject.) }\end{array}$ & $15 \mu \mathrm{A}$ & $15.8 \mu \mathrm{A}$ \\
$\begin{array}{l}\text { Current consumption } \\
@ V_{D D}=3 \mathrm{~V}\end{array}$ & & \\
\hline
\end{tabular}

${ }^{*}$ Limited by breakdown.

Table 2. Performance comparison of this optimized simple voltage reference circuit with three other precise voltage reference circuits reported in the literature.

\begin{tabular}{lcccc}
\hline & \multirow{2}{*}{$\begin{array}{c}I_{\text {Diss }} \\
\text { Case }\end{array}$} & $\begin{array}{c}\text { Input } \\
\text { range }\end{array}$ & Static & Dynamic \\
\cline { 4 - 5 } & & & \multicolumn{2}{c}{ Line regulation } \\
\hline$[9]$ & 8.6 & $1.3-3 \mathrm{~V}$ & $53 \mathrm{~m} \mathrm{~V} / \mathrm{V}$ & - \\
{$[10]$} & $7 @ 3.5^{V}$ & $1.3-3.5 \mathrm{~V}$ & $45 \mathrm{mV} / \mathrm{V}$ & $34 \mathrm{~dB}$ \\
{$[11]$} & $89 @ 5^{V}$ & $3.3-7 \mathrm{~V}$ & $6.2 \mathrm{mV} / \mathrm{V}$ & $43.2 \mathrm{~dB}$ \\
& & & & $@ 1 \mathrm{kHz}$ \\
This & $18.4 @ 5^{V}$ & $2.6-12 \mathrm{~V}^{*}$ & $957 \mu \mathrm{V} / \mathrm{V}$ & $60 \pm 3 \mathrm{~dB}$ \\
work & & & & $@ f \leq 8 \mathrm{MHz}$
\end{tabular}

*Limited by breakdown. demonstrate great performance for a wide-range supply-independent voltage reference both in simulations and experiments. Table 2 compares this optimized voltage reference circuit with three other precise voltage reference circuits reported in the literature. It is noted that the circuit reported here provides the best performance in terms of voltage stability (regulation), power consumption, and ripple rejection of all the circuits so far reported.

\subsection{Multi-Output Voltage Reference}

Shown in Fig. 12 is the photo of the proposed voltage reference circuit along with its startup circuit that has been fabricated using MOSIS 1.6 um standard CMOS process.

Figure 13 shows the DC transfer curves of the optimized reference circuit obtained from simulations and experiments for the main reference output. Again, the input-output characteristic curve is still extremely flat over a wide range of input from about 6 to $12 \mathrm{~V}$. However simulations show that the flatness of the DC transfer curve continues up to input voltages as high as 20 V (Fig. 7) and even higher, we couldn't apply input voltages above $12 \mathrm{~V}$ (in some cases $14 \mathrm{~V}$ ) since the transistors were burned out.

Table 3. Simulated and measured specifications of the multi-output voltage reference circuit.

\begin{tabular}{lcc}
\hline Specification & Simulated & Measured \\
\hline Input voltage range & $5-20 \mathrm{~V}$ & $6-14 \mathrm{~V}^{*}$ \\
$\begin{array}{l}\text { Static supply } \\
\quad \text { dependency (DC) }\end{array}$ & $1.53 \mathrm{mV} / \mathrm{V}$ & $1.33 \mathrm{mV} / \mathrm{V}$ \\
$\begin{array}{l}\text { Dynamic supply } \\
\quad \text { dependency (Ripple reject.) }\end{array}$ & $-52.8 \mathrm{~dB}$ & $-48 \mathrm{~dB}$ \\
$\begin{array}{l}\text { Current consumption } \\
\quad \text { voltage ref. \& startup circuit) }\end{array}$ & $\begin{array}{l}23 \mathrm{uA} \\
\text { @ } 20 \mathrm{~V} \text { input }\end{array}$ & $25 \mathrm{uA}$ \\
\hline
\end{tabular}

*Limited by breakdown.

Table 4. Performance comparison of this optimized multi-output voltage reference circuit with three other precise voltage reference circuits reported in the literature.

\begin{tabular}{lcccc}
\hline & & Input & \multicolumn{2}{c}{ Line regulation } \\
\cline { 4 - 5 } Case & $P_{\text {Diss }}$ & range & Static & Dynamic \\
\hline$[9]$ & $8.6 \mathrm{uA}$ & $1.3-3 \mathrm{~V}$ & $53 \mathrm{mV} / \mathrm{V}$ & - \\
{$[10]$} & $7 \mathrm{uA}$ & $1.3-3.5 \mathrm{~V}$ & $45 \mathrm{mV} / \mathrm{V}$ & $34 \mathrm{~dB}$ \\
& $@ 3.5^{V}$ & & & \\
{$[11]$} & $89 \mathrm{uA}$ & $3.3-7 \mathrm{~V}$ & $6.2 \mathrm{mV} / \mathrm{V}$ & $43.2 \mathrm{~dB}$ \\
& $@ 5^{V}$ & & $01 \mathrm{kHz}$ & \\
This & $25 \mathrm{uA}$ & $6-14 \mathrm{~V}$ & $1.33 \mathrm{mV} / \mathrm{V}$ & $48-57.4 \mathrm{~dB}$ \\
work & & & & $@ f \leq 1 \mathrm{MHz}$ \\
\hline
\end{tabular}

*Limited by breakdown. 


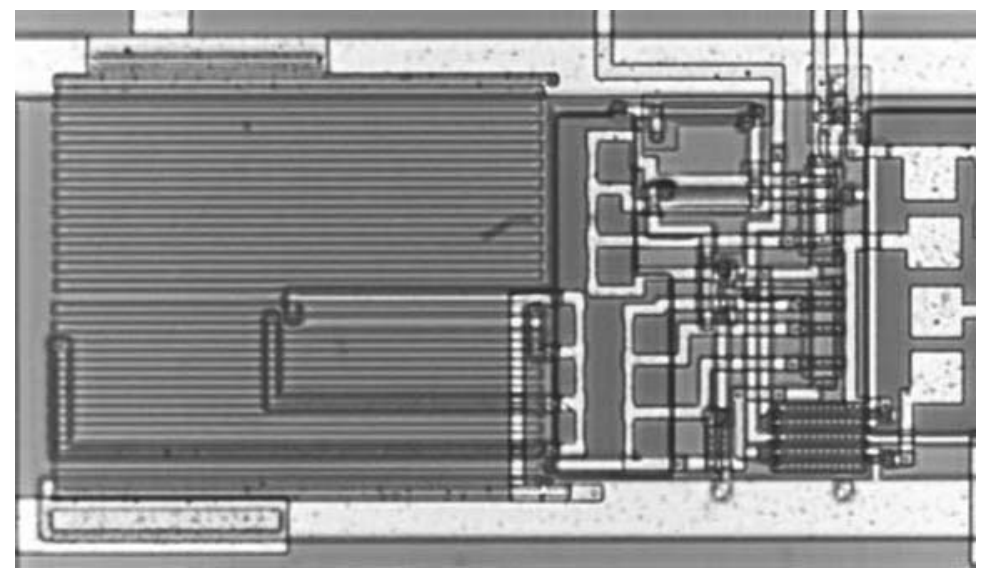

Fig. 12. Photo of the proposed voltage reference circuit along with its startup circuit.

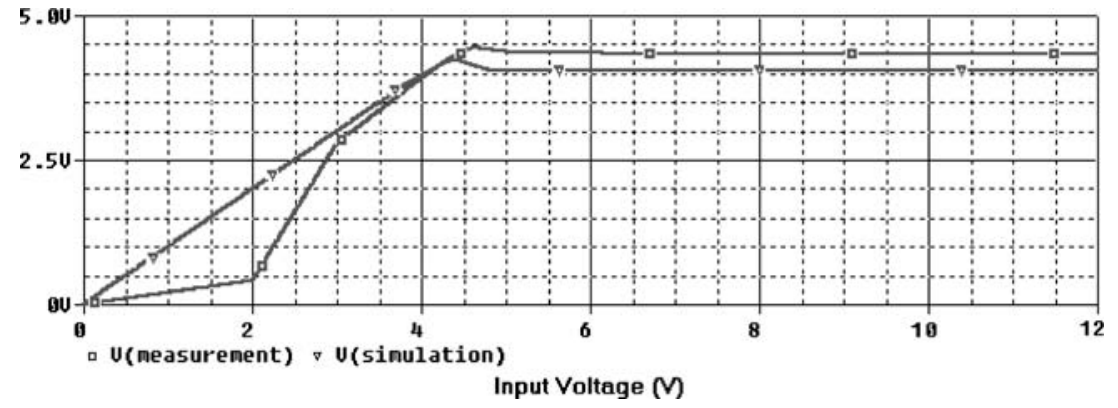

Fig. 13. DC transfer curves for the circuit, resulted from simulation and measurement.

Measurements show that the ripple rejection ratio of the circuit has fallen from $-52.8 \mathrm{~dB}$ in simulations to $-48 \mathrm{~dB}$.

Table 3 summarizes the specification of the voltage reference circuit obtained from both simulations and measurements. Table 4 compares the optimized voltage reference circuit with three other precise voltage reference circuits.

\section{Conclusion}

A voltage reference circuit has been designed in standard CMOS process that exhibits excellent supply independency. This supply independency is well observed in the static operation of the circuit over an extremely wide input range, and also in its dynamic behavior for high frequency ripples on the input voltage. The circuit, fabricated using MOSIS $1.6 \mu \mathrm{m}$ standard CMOS process, shows $957 \mathrm{uV} / \mathrm{V}$ static supply dependency, $60 \mathrm{~dB}$ ripple rejection, and consumes 15.8 to $36.9 \mathrm{uA}$ when the input voltage varies from 2.6 to $12 \mathrm{~V}$. This $\mathbf{1 2 - V}$ maximum input is just the breakdown limitation that didn't let the measurements go farther, but based on the close agreements observed between simulation and experimental results, the circuit is expected to continue its highly supply independent operation for up to $20-\mathrm{V}$ input.
Based on the proposed idea, a multi-output voltage reference and a CMOS DC level shifter are also designed.

\section{Appendix}

From the circuit we have:

$$
\begin{aligned}
V_{G S 1} & =V_{T N}+\left(2 I_{1} / \beta_{1}\right)^{1 / 2} \\
V_{G S 2} & =V_{T N}+\left[2 I_{R} / \beta_{2}\left(1+\lambda_{N} V_{D S 2}\right)\right]^{1 / 2} \\
I_{1} & =I_{R} \cdot\left[1+\lambda_{P} V_{S D 3}\right] \\
I_{R} & =V_{G S 1} / R
\end{aligned}
$$

Considering (3), and also approximating $\delta V_{D S 2} /$ $\delta V_{D D}$ to 1 for more simplification, we will have:

$$
\begin{aligned}
& \left(\delta V_{G S 1} / \delta V_{D D}\right) \cdot\left[\sqrt{2 b \beta_{1} / I_{R}}-b /\left(R I_{R}\right)\right]=\lambda_{P} \\
& \sqrt{2 a^{3} \beta_{2} R V_{G S 1}}\left(\delta V_{G S 2} / \delta V_{D D}\right) \\
& \quad=\left[a \cdot\left(\delta V_{G S 1} / \delta V_{D D}\right)-\lambda_{N} V_{G S 1}\right]
\end{aligned}
$$

where $a=1+\lambda_{N} V_{D S 2}$ and $b=1+\lambda_{P} V_{S D 3}=1+$ $\lambda_{P}\left(V_{D D}-V_{\mathrm{REF}}\right)$. 
Now, in order to have a supply-independent reference voltage, Eq. (4) helps us to rewrite (A6) as:

$$
\left(\delta V_{G S 1} / \delta V_{D D}\right)\left(a+\sqrt{\left.2 a^{3} \beta_{2} R V_{G S 1}\right)}=\lambda_{N} V_{G S 1}\right.
$$

and using (A4), (A7) can be rewritten as:

$$
\left(\delta V_{G S 1} / \delta V_{D D}\right) \cdot\left(a / R I_{R}+\sqrt{\left.2 a^{3} \beta_{2} / I_{R}\right)}=\lambda_{N}\right.
$$

Dividing (A8) by (A5) and rearranging the resulted equation leads to:

$$
\begin{aligned}
& a / R I_{R}+\left(\lambda_{N} / \lambda_{P}\right) b / R I_{R} \\
& =\left(\lambda_{N} / \lambda_{P}\right)\left(2 b \beta_{1} / I_{R}\right)^{1 / 2}-\left(2 a^{3} \beta_{2} / I_{R}\right)^{1 / 2}
\end{aligned}
$$

and

$$
\begin{aligned}
a+\left(\lambda_{N} / \lambda_{P}\right) b= & R\left(2 I_{R}\right)^{1 / 2}\left[\left(\lambda_{N} / \lambda_{P}\right)\left(b \beta_{1}\right)^{1 / 2}\right. \\
& \left.-\left(a^{3} \beta_{2}\right)^{1 / 2}\right]
\end{aligned}
$$

and eventually:

$$
\left(R \sqrt{2 I_{R}}\right)\left(\lambda_{N} \sqrt{b \cdot \beta_{1}}-\lambda_{P} \sqrt{a^{3} \beta_{2}}\right)=b \lambda_{N}+a \lambda_{P}
$$

In order to introduce (A.10) as a design equation, $a$ and $b$ should be defined in term of known quantities. So,

$$
\begin{aligned}
a & =1+\lambda_{N} V_{D S 2} \\
& =1+\lambda_{N}\left(V_{D D}-V_{S G 4}-V_{G S 1}\right) \\
& =1+\lambda_{N}\left(V_{D D}-V_{\mathrm{REF}}\right)+\lambda_{N}\left(V_{G S 2}-V_{S G 4}\right) \\
& \cong 1+\lambda_{N}\left(V_{D D}-V_{\mathrm{REF}}\right) \\
b & =1+\lambda_{P} V_{S D 3}=1+\lambda_{P}\left(V_{D D}-V_{\mathrm{REF}}\right)
\end{aligned}
$$

\section{Acknowledgments}

This work was supported primarily by the Engineering Research Centers Program of the National Science Foundation under Award Number EEC-9986866.

\section{References}

1. B. Smith et al., "An externally powered, multichannel, implantable stimulator-telemeter for control of paralyzed muscle," IEEE Transactions on Biomedical Engineering, vol. 45, no. 4, pp. 463-475, 1998.

2. T. Akin et al., "A wireless implantable multichannel digital neural recording system for a micromachined sieve electrode," IEEE Journal of Solid-State Circuits, vol. 33, no. 1, pp. 109-118, 1998.
3. B. Ziaie et al., "A single channel implantable microstimulator for functional neuromuscular stimulation," IEEE Transactions on Biomedical Engineering, vol. 44, no. 10, pp. 909-920, 1997.

4. M. Nardin and K. Najafi, "A multichannel neuromuscular microstimulator with bi-directional telemetry," in Proceedings of the 1995 Transducers Conference, Sweden, 1995.

5. G. Gudnason et al., "A chip for an implantable neural stimulator," Analog Integrated Circuits and Signal Processing, Kluwer Academic Publishers, vol. 22, pp. 81-89, 1999.

6. P.E. Allen and D.A. Holberg, CMOS Analog Circuit Design, 2 nd Edition, Oxford University Press, 2002.

7. A.M. Sodagar and K. Najafi, "A wide-range supply-independent CMOS voltage reference for telemetry-powering applications," in Proceedings of the 2002 IEEE International Conference on Electronics, Circuits, and Systems (ICECS'02), pp. 401-404.

8. A.M. Sodagar and Khalil Najafi, "A multi-output supplyindependent voltage reference in standard cmos process for telemetry-powering applications," in Proceedings of the 2003 IEEE International Symposium on Signals, Circuits, and Systems, pp. 493-496.

9. T. Kawahara et al., "Internal voltage generator for low-voltage quarter-micrometer flash memories," IEEE Journal of Solid-State Circuits, vol. 33, no. 1, pp. 126-132, 1998.

10. H. Tanaka et al., "A precise on-chip voltage generator for a gigascale DRAM with a negative word-line scheme," IEEE Journal of Solid-State Circuits, vol. 34, no. 8, pp. 1084-1090, 1999.

11. W.T. Harrison, J.A. Connelly, and R. Stair, "An improved currentmode CMOS voltage reference," in Proceedings of the 2001 Southwest Symposium on Mixed-Signal Design (SSMSD'2001), pp. 23-27.

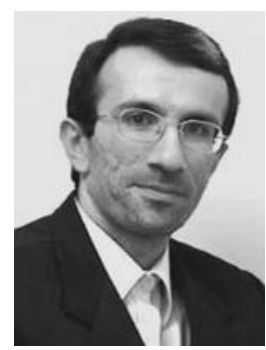

Amir M. Sodagar received the B.S. degree from K.N. Toosi (KNT) University of Technology, Tehran, Iran, and M.S. and Ph.D. degrees from Iran University of Science \& Technology (IUST), Tehran, Iran all in Electrical Engineering in 1992, 1995, and 2000, respectively. From 1992 to 2000 he was with S. Rajaee University as a Lecturer. After receiving the Ph.D. degree until 2002 he was with the NSF Engineering Research Center for Wireless Integrated Micro Systems (WIMS), Electrical Engineering \& Computer Science (EECS) Dept., University of Michigan as a Post-Doctoral Research Fellow. From 2002 to 2004 he was with S. Rajaee University and KNT University of Technology as an Assistant Professor and an Adjunct Professor, respectively, and since 2004 he has been with the University of Michigan as an Associate Visiting Research Scientist.

Dr. Sodagar was known as the Outstanding Electrical Engineering Graduate Student of the IUST in 1995, and received the IUST's Best Ph.D. Research Achievement 
Award in 2000. He was also the recipient of S. Rajaee University's Distinguished Faculty Member Award for "1998-1999" and "1999-2000" academic years, and S. Rajaee University's Distinguished Researcher Award for "2002-2003" academic year.

He was involved in the design of integrated circuits in collaboration with the Center for Semiconductor Research and Fabrication from 1994 to 1995, VLSI Circuits \& Systems Laboratory at the University of Tehran from 1997 to 1998, and EMAD Semicon Company from 1998 to 2000. He has authored one book, authored/co-authored more than 20 journal and conference papers, and served as the technical paper reviewer for several IEEE journals/transactions and also conferences.

Dr. Sodagar's research interests are generally in the field of mixed-signal integrated circuit design, and focused on: integrated circuits for neural recording \& stimulation, telemetry powering and control of implantable microsystems, frequency synthesizers, analog building blocks, and transistor-level implementations of digital logic families.

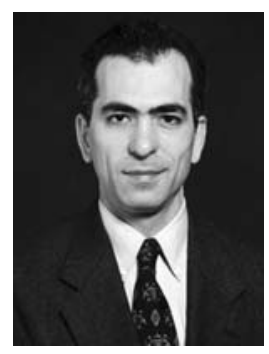

Khalil Najafi (IEEE S '84, M '86, SM '97, F'00) received the B.S., M.S., and the Ph.D. degree in 1980, 1981, and 1986 respectively, all in Electrical Engineering from the Department of Electrical Engineering and Computer Science, University of Michigan, Ann Arbor. From 1986-1988 he was employed as a Research Fellow, from 1988-1990 as an Assistant Research Scientist, from 1990-1993 as an Assistant Professor, from 1993-1998 as an Associate Professor, and since September 1998 as a Professor and the Director of the Solid-State Electronics Laboratory, Department of Electrical Engineering and Computer Science, University of Michigan. His research interests include: micromachining technologies, micromachined sensors, actuators, and MEMS; analog integrated circuits; implantable biomedical microsystems; micropackaging; and low-power wireless sensing/actuating systems.

Dr. Najafi was awarded a National Science Foundation Young Investigator Award from 1992-1997, was the recipient of the Beatrice Winner Award for Editorial Excellence at the 1986 International Solid-State Circuits Conference, of the Paul Rappaport Award for coauthoring the Best Paper published in the IEEE Transactions on Electron Devices, and of the Best Paper Award at ISSCC 1999. In 2003 he received the EECS Outstanding Achievement Award, in 2001 he received the Faculty recognition Award, and in 1994 the University of Michigan's "Henry Russel Award" for outstanding achievement and scholarship, and was selected as the "Professor of the Year" in 1993. In 1998 he was named the Arhtur F. Thurnau Professor for outstanding contributions to teaching and research, and received the College of Engineering's Research Excellence Award. $\mathrm{He}$ has been active in the field of solid-state sensors and actuators for more than twenty years, and has been involved in several conferences and workshops dealing with solid-state sensors and actuators, including the International Conference on Solid-State Sensors and Actuators, the Hilton-Head Solid-State Sensors and Actuators Workshop, and the IEEE/ASME Micro Electromechanical Systems (MEMS) Conference. Dr. Najafi is the Editor for Solid-State Sensors for IEEE Transactions on Electron Devices, an Associate Editor for the Journal of Micromechanics and Microengineering, Institute of Physics Publishing, and an editor for the Journal of Sensors and Materials. He also served as the Associate Editor for IEEE Journal of Solid-State Circuits from 2000-2004, and the associate editor for IEEE Trans. Biomedical Engineering from 1999-2000. He is a Fellow of the IEEE. 